\title{
Indirect Estimation of Clinical Chemical Reference Intervals from Total Hospital Patient Data: Application of a Modified Bhattacharya Procedure
}

\author{
By H. Baadenhuijsen and J. C. Smit \\ Laboratory of Clinical Chemistry, Department of Internal Medicine, St. Radboud University Hospital, Nijmegen, \\ The Netherlands
}

(Received February 22/June 12, 1985)

\begin{abstract}
Summary: Indirect methods for the determination of reference intervals utilise as input the total bulk of test results from all admitted hospital patients. One of the strongest arguments in favour of the use of these indirect methods is that one automatically obtains a one to one relationship between the selected population sample and the category of people for which the test results are intended. A study was conducted of the suitability of a modification of the indirect Bhattacharya method, which also permits the evaluation of heavily skewed distributions. The robustness of the method was evaluated by studying the homogeneity, stability and reproducibility of the hospital population in terms of the statistical parameters of the input distributions and of the resulting reference intervals for creatinine, uric acid and alanine aminotransferase. Finally, the age- and sex dependency for 15 clinical chemical parameters was compared with reported literature data.
\end{abstract}

Indirekte Bestimmung klinisch-chemischer Referenzintervalle aus den gesamten Daten von Klinikpatienten: Anwendung eines modifizierten Bhattacharya-Verfahrens

Zusammenfassung: Indirekte Methoden für die Bestimmung von Referenzintervallen verwenden als Eingabe die Gesamtheit der Testergebnisse von allen in die Klinik eingewiesenen Patienten. Eines der stärksten Argumente für die Anwendung dieser indirekten Methoden ist, daß von selbst eine eins-zu-eins-Beziehung zwischen der ausgewählten Populationsstichprobe und der Probandenkategorie, für die die Testergebnisse bestimmt sind, erhalten wird. Die Eignung einer Modifikation der indirekten Bhattacharya-Methode, die auch die Auswertung von sehr schiefen Verteilungen gestattet, wurde geprüft. Durch Untersuchungen der Homogenität, Stabilität und Reproduzierbarkeit der Patientenpopulation hinsichtlich der statistischen Kenngrößen der Eingabeverteilungen und der erhaltenen Referenzintervalle für Kreatinin, Harnsäure und Alaninaminotransferase wurde die Robustheit der Methode geprüft. Schließlich wurde die Alters- und Geschlechtsabhängigkeit von 15 klinisch-chemischen Kenngrößen mit Literaturdaten verglichen.

\section{Introduction}

It is now generally accepted that the term "Normal Values" should be abolished when dealing with the criterion for the intuitive interpretation of test results. The application of a normal range can only be justified when a representative and homogeneous selection of the healthy population is present for the prior study of its "healthy" parameters. The problems associated with ascertaining state of health are great.
Therefore we agree with Schütz (1) that it is often easier to diagnose illness than health.

Considerations regarding the semantics of the reference interval concept has been discussed at length by several authors $(2-6)$. As advocated by the IFCC Expert Panel on Theory of Reference Values (7) it is good practice to speak of reference values that result in a reference interval after appropriate assessment 
of the tails of the observed distributions. It is also necessary that the reference interval is preceded by a qualifying epithet describing the way the specimens were treated and analysed.

Because of the problems encountered in selecting the "healthy" target group attempts have been made in the past to determine reference intervals from the total bulk of unselected results that are daily produced in the clinical laboratory $(8-11)$. One of these so-called indirect methods uses the principles described by Bhattacharya (12). The algorithm assumes that the major part of the total unselected population can be considered "normal" and that the overlap between this "healthy" part and the abnormal (high and/or low) part is only partial.

As can be seen from the underlying mathematics, the decision as to whether or not the main distribution can be resolved is easily made when the healthy distribution in question is indeed Gaussian. For those distributions that are not Gaussian, the standard algorithm will fail. Hereafter we propose a modification of the Bhattacharya procedure which also makes it possible to deal with skewed distributions.

\section{Methods}

\section{Mathematical}

Use was made of the computer software package REFVALUE which was developed by us and made commercially available (13). The statistical approach involved the use of a relatively simple procedure for the resolution of overlapping Gaussian distributions (12). The Gaussian or normal probability density function $\mathrm{Y}(\mathrm{x})$ can be defined as

$$
\mathrm{Y}(\mathrm{x})=\frac{1}{\sigma \sqrt{2 \pi}} \mathrm{e}^{-(\mathrm{x}-\mu)^{2} / 2 \sigma^{2}}
$$

where $\mu$ and $\sigma>0$ are respectively the mean and standard deviation of the distribution. The peak-shaped probability density function can be linearised in two steps: applying a log conversion for the $\mathrm{Y}$-values (eq. 2), and obtaining a first derivative after $x$ (eq. 3 ):

$$
\begin{aligned}
& \ln (Y(x))=\ln \left[\frac{1}{\sigma \sqrt{2 \pi}}\right]-(x-\mu)^{2} / 2 \sigma^{2} \\
& \frac{d \ln (Y(x))}{d x}=-\frac{1}{\sigma^{2}} x+\mu / \sigma^{2}
\end{aligned}
$$

By plotting $d \ln (Y(x)) / d x$ against $x$ a straight line is obtained with slope $-1 / \sigma^{2}$ and intercept $\mu / \sigma^{2}$. After replacing the differential operator by a forward difference operator $\Delta$, this yields:

$$
\frac{\Delta \ln Y i(x i)}{\Delta x}=-\frac{1}{\sigma^{2}} x i+\mu / \sigma^{2}
$$

This means that by plotting $\Delta \ln (Y)$ against $x$, the Gaussian component of the total distribution will be visualised, if it is present, by the display of the linear part of the first derivative function. Proper locating of this linear part with its slope and abscissa-intercept results in the processing of these parameters into the estimated values of the mean and standard deviation. This is illustrated in figure 3 for the creatinine results (men, born between 1940 and 1950). The values in figure 3 stem from the original distribution as depicted in 'figures 1 and 2 as a frequency distribution. Thus calculation of $\mathrm{A}$ and $\mathrm{B}$ in the formula $\mathrm{Y}=\mathrm{A}+\mathrm{Bx}$ using a least squares method results in:

Estimated value for the mean, $\mu$ : $-\mathrm{A} / \mathrm{B}$ and estimated value for the variance, $\sigma^{2}:-1 / B$.

To adjust for the grouping error a correction term $h^{2} / 12$ (Shephards correction) has to be subtracted from the calculated value of $\sigma^{2}$, and a correction term $h / 2$ has to be added to the calculated value of $\mu$, where $h$ equals the class-width in the grouping procedure. The reference interval is finally calculated as the interval covered by the $2 * 1.96$ times the standard deviation range left and right from the calculated mean value.

In those cases where all observations belong to one particular population sample, relatively simple statistical tests exist $\left[\chi^{2}\right.$ test. Kolmogorov-Smirnov test (see 1. c. (14)), Shapiro \& Wilk test (15)] to prove or disprove that a distribution is Gaussian. Using indirect methods, with observations not belonging to one particular distribution, such tests are not feasible. However, a good indication of whether one is dealing with an underlying non-Gaussian subpopulation can be obtained from the nonlinearity in the Bhattacharya plot of the first derivative function. This is illustrated in figure 9 for the $\gamma$-glutamyltransferase results of men (born between 1940 and 1950) where, in the region between 14 and $25 \mathrm{U} / 1$, a linear correlation coefficient of only 0.878 was obtained. Two alternative ways of approaching this sort of distributions seem to be possible. One of these is the assumption that the underlying normal population can be described by a Gamma function $(3,10)$. The other approach is to transform the observed frequencies along the abscissa by some mathematical function (16-19). A frequently used function is $Z=\ln (x+c)$, where $c$ is a transformation constant, which can be iteratively computed to produce a transformed function with a skewness of zero. Application of this transformation was advocated by Harris \& DeMets (18). From their work it became also apparent that minimizing the kurtosis improved the estimators only very slightly or not at all. Using this $\ln (x+c)$ transformation on non-selected patient data introduces the problem that the transformation parameter, $c$, cannot be determined on the basis of the total distribution. It should operate only upon that part of the distribution that represents the underlying nondiseased part of the population. This part of the program gives the opportunity to choose the region of interest to be transformed. Figure 8 gives the smoothed input distribution of the results depicted in figure 7 , together with the chosen setting of a boundary which intersects the distribution roughly at the points 14 and $25 \mathrm{U} / \mathrm{l}$ covering the region which gave the nearly linear relation as shown in figure 9. The result of the transformation procedure gives now a symmetrical central portion of the total distribution and is shown in figure 10 . Note that the abscissa units now appear on a logarithmic scale. Further processing of this transformed function is illustrated in figures 11 and 12 . In figure 11 there is now a strict linear relation in the first derivative function, with a linear correlation coefficient of 0.999 between the abscissa values 9 and $22 \mathrm{U} / 1$. From the slope and abscissa intercept, the calculated distribution is thereafter generated with help of the known analytical function:

$$
\mathrm{Yi}=\frac{1}{\sigma \sqrt{2 \pi}} \mathrm{e}^{-(\mathbf{x i}-\mu)^{2} / 2 \sigma^{2}}
$$

using the estimated value for $\mu$ and $\sigma^{2}$. 


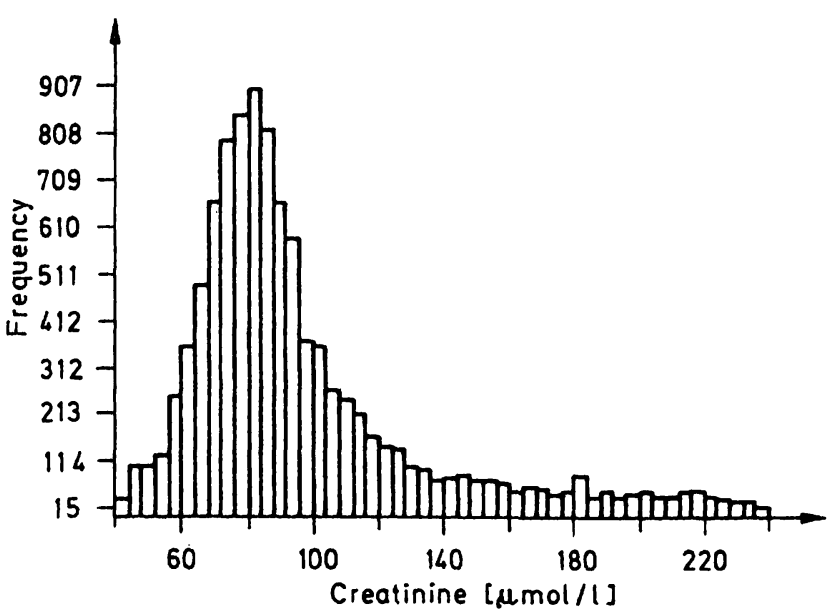

Fig. 1. Total input distribution of serum creatinine from males born between 1940 and 1950 .

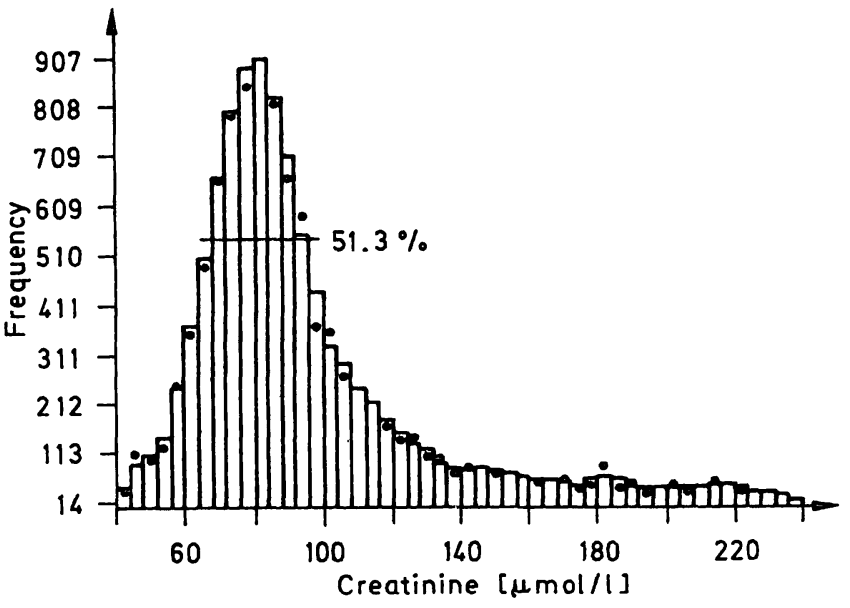

Fig. 2. Distribution as in figure 1 (dotted) and after applying a five-point Savitsky \& Golay smoothing procedure (solid lines). Also shown is the intersecting frequency level for application of a transformation procedure.

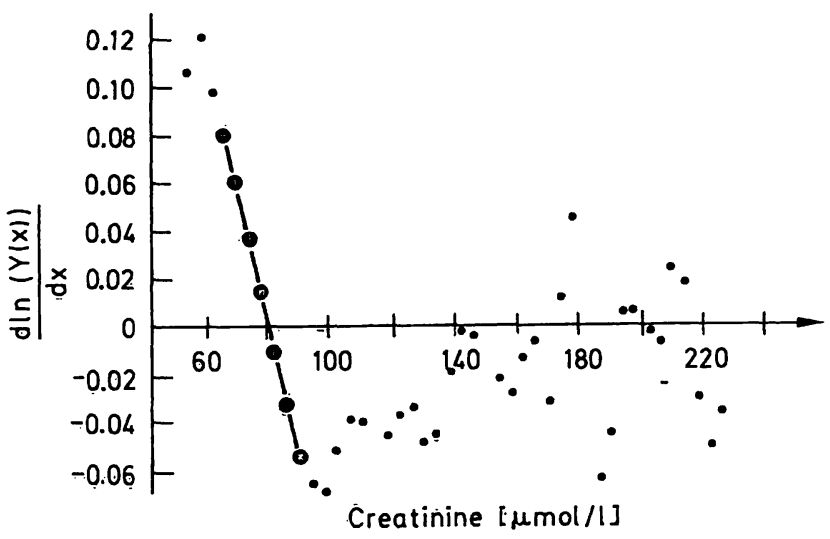

Fig. 3. Plot of the first derivative function for the creatinine results. No transformation has been applied in this case. Good linearity (correlation coefficient of 0.999 ) is revealed in the region between 65 and $95 \mu \mathrm{mol} / \mathrm{l}$.

For a lognormal distribution, as was the case in the abovementioned $\gamma$-glutamyltransferase example, this formula reads:

$$
Y i=\frac{1}{\sigma \sqrt{2 \pi}} e^{-(\ln (x i+c)-\mu)^{2} / 2 \sigma^{2}},
$$

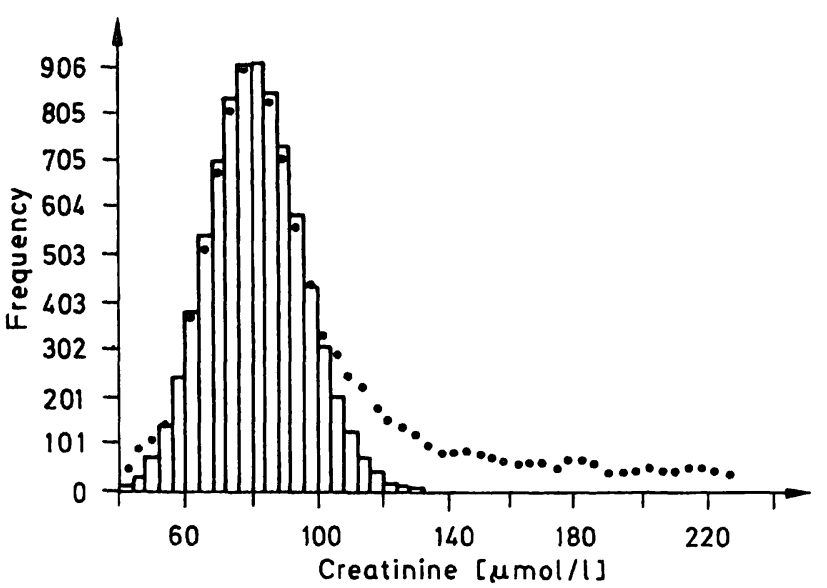

Fig. 4. Plot of the calculated distribution (solid lines) with a corresponding reference interval of $53.4-107.6 \mu \mathrm{mol} / \mathrm{l}$ for the creatinine results, displayed together with the original smoothed input distribution (dotted).

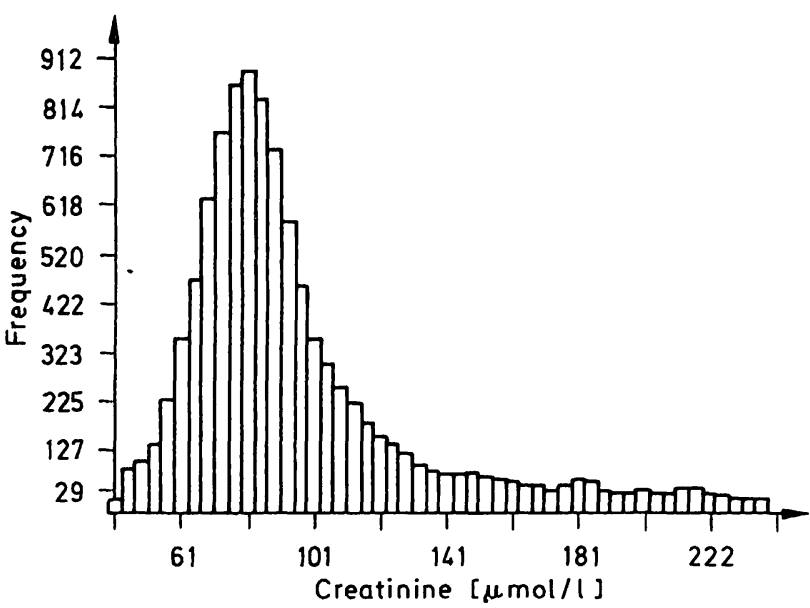

Fig. 5. Plot of the input creatinine distribution after transformation with the function $Z=\ln (x+c)$, c being 3513.45 , on the $51.3 \%$ proportion of the total distribution as depicted in figure 2 .

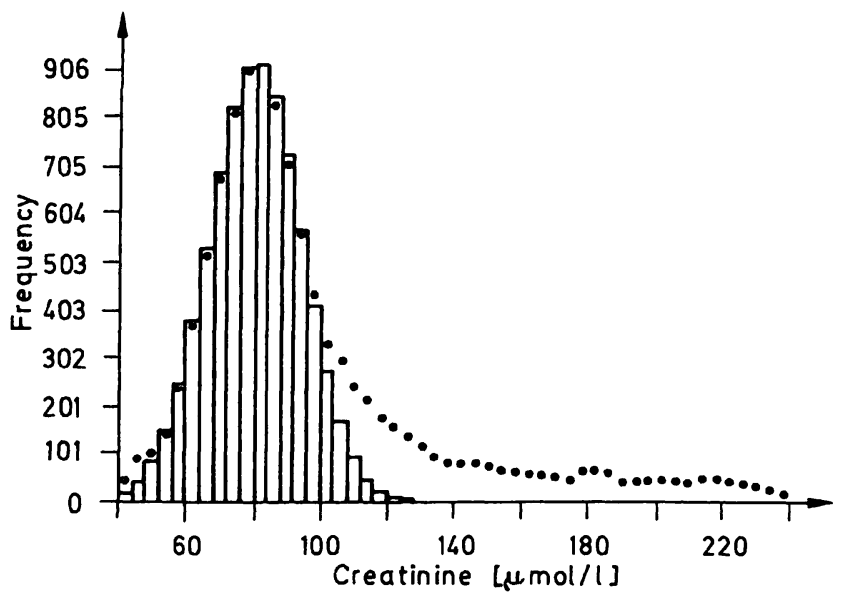

Fig. 6. Plot of the calculated distribution (solid lines) with a corresponding reference interval of $53.8-108.2 \mu \mathrm{mol} / \mathrm{l}$, together with the original distribution (dotted) after application of the transformation procedure.

in which $\mathrm{c}$ is the calculated transformation constant in $\mathrm{Z}=$ $\ln (x+c)$, which amounted 2.022 as illustrated in figure 10 . Finally, this calculated distribution, with reference interval of $6.6-34.8 \mathrm{U} / \mathrm{l}$, can be displayed together with the original total distribution as shown in figure 12. 


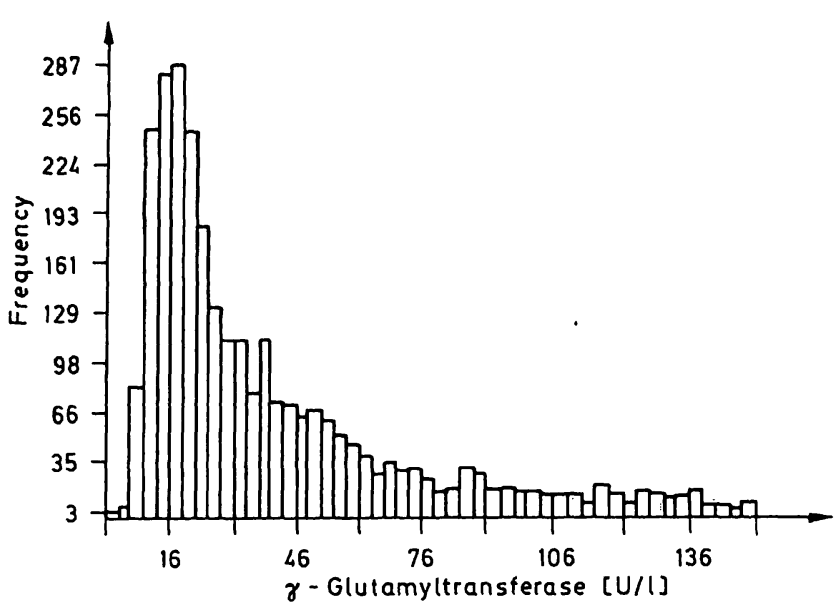

Fig. 7. Total input distribution of serum $\gamma$-glutamyltransferase from males born between 1940 and 1950 .

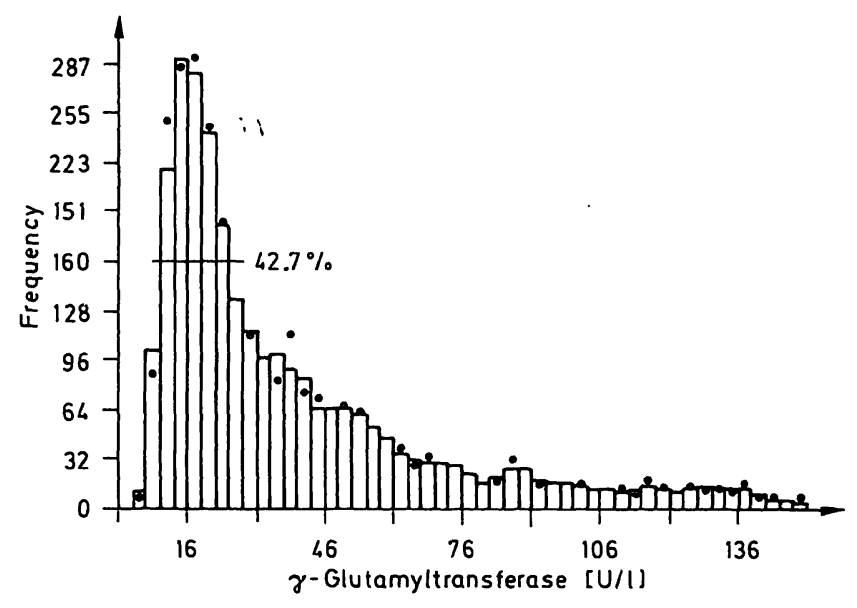

Fig. 8. Distribution as in figure 7 (dotted) and after applying a five-point Savitsky \& Golay smoothing procedure (solid lines). Also shown is the intersecting frequency level for application of the transformation procedure.

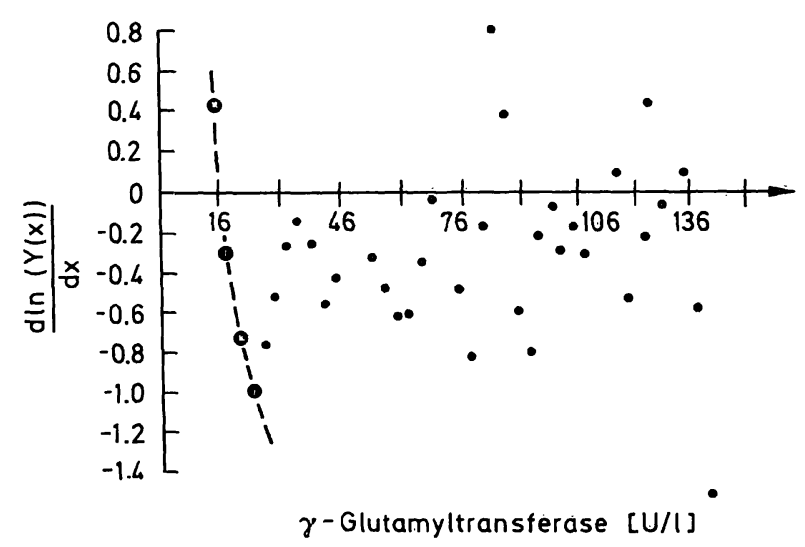

Fig. 9. Plot of the first derivative function for the $\gamma$-glutamyltransferase results. No transformation has been applied in this case. The result is a far from perfect linear portion (correlation coefficient of 0.878 ) between 14 and $25 \mathrm{U} / 1$.

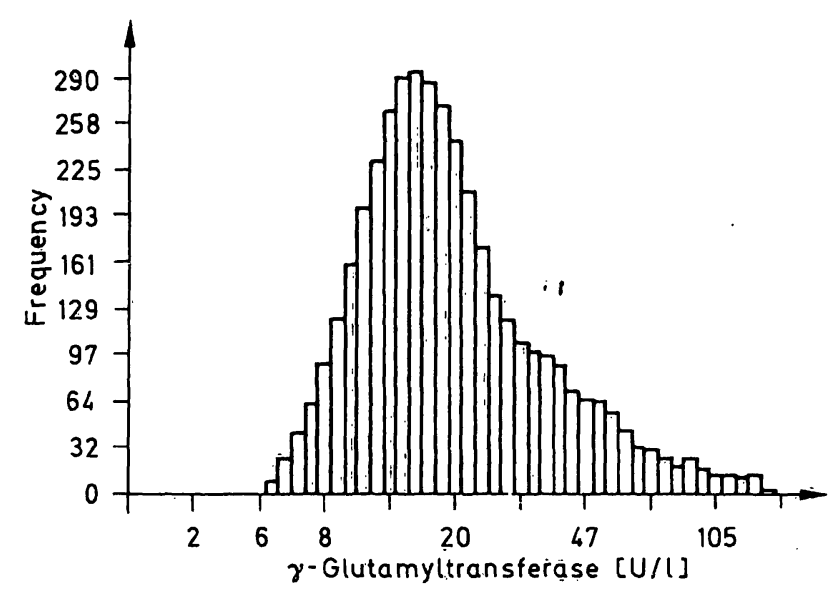

Fig. 10. Plot of the input $\gamma$-glutamyltransferase distribution after transformation with the function $Z=\ln (x+c)$, c being 2.022 , on the $42.7 \%$ proportion of the total distribution as depicted in figure 8 .

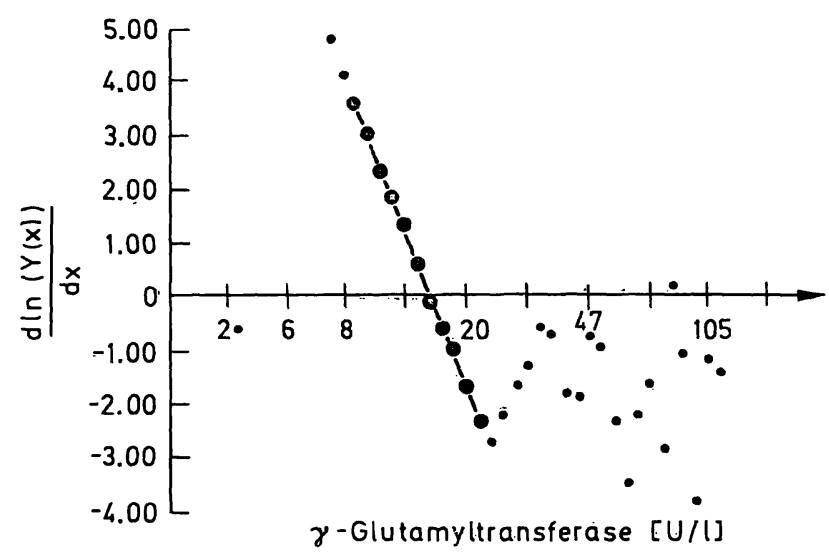

Fig. 11. Plot of the first derivative function for the $\gamma$-glutamyltransferase results after transformation. In comparison with figure 9 the highly improved linearity (correlation coefficient of 0.999 ) between 9 and $22 \mathrm{U} / \mathrm{l}$ is obvious.

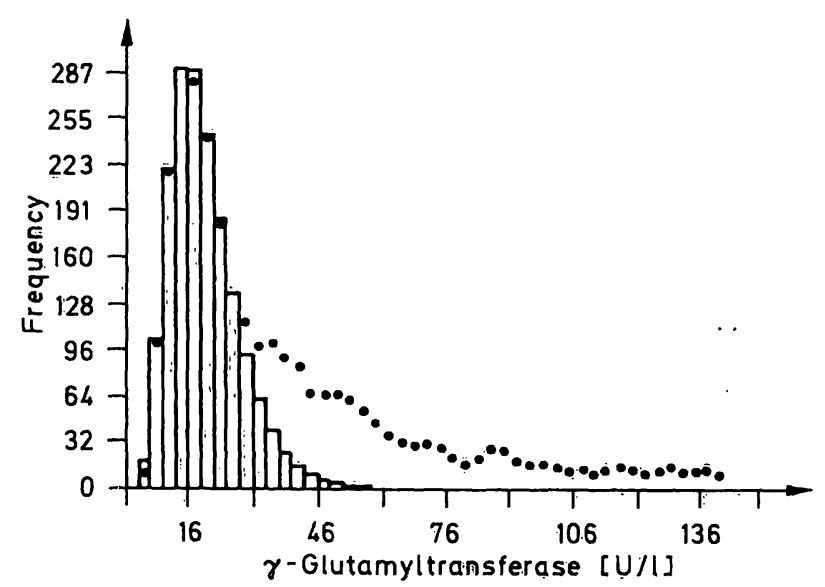

Fig. 12. Plot of the calculated distribution (solid lines) with a corresponding reference interval of $6.6-34.8 \mathrm{U} / \mathrm{l}$ for the $\gamma$-glutamyltransferase results, together with the original smoothed distribution (dotted) after application of the transformation procedure. 


\section{Procedural}

The execution of the program is based on the prior construction of sequential disc files containing the various test results; the $x$-array containing equidistant concentration (or activity) intervals and the Y-array the cumulated frequencies in each class interval. One of the prerequisites for a proper application of the Bhattacharya deconvolution technique is the absence of large statistical fluctuations in the frequency distribution that prevents recognition of the linear part of the first derivative function. It is therefore often necessary to accumulate over 1500 values for each parameter to be studied. In order to further enlighten the later statistical evaluations, a filter algorithm according to Savitsky \& Golay (20) as advocated by Naus (11) has been included in the program. Results of this smoothing procedure are given in figures 2 and 8 for the creatinine and $\gamma$-glutamyltransferase examples.

\section{Chemical}

In view of the scope of this study no benefit from a listing of the chemical methods used for the determination of the various constituents was expected and has therefore been omitted.

\section{Results}

Our main concern was to evaluate the efficiency of the transformation algorithm and to explore the possibility of performing the transformation routine also in those cases where the healthy subpopulation appeared to be Gaussian in nature, as detected from an existing linear relationship in the first derivative function. It was thought that a consequent application of the transformation procedure would enable a more straightforward evaluation of all existing result files. In figures $2-6$ the comparison of the two alternative procedures on the serum creatinine results from men born between 1940 and 1950 depicted in figure 1, is illustrated. In figures 3 and 4 the results are processed directly, without prior transformation, and in figures 5 and 6 with inclusion of the transformation algorithm. Direct processing results in a reference interval of $53.4-107.6 \mu \mathrm{mol} / 1$. Direct processing appears justified because of the existing clearcut linearity of the first derivative function between 65 and $95 \mu \mathrm{mol} / 1$ as can be seen in figure 3 . In figure 2 the proportion of the total population which is thereafter processed in the transformation procedure is depicted as $51.3 \%$. This value resulted from choosing the setting of the boundary intersecting the total distribution roughly at the points 65 and $95 \mu \mathrm{mol} / \mathrm{l}$, covering the linear part of the first derivative function as processed directly. In figure 5 the appearance of the total distribution is given after being processed by the function $Z=\ln (x+c)$, c being 3513.45. Comparison of figures 2 and 5 reveals the close resemblance of these two distributions in terms of the abscissa values with their associate frequency values. Again, from the slope and intercept of the linear part of the first derivative function (not shown), the calculated distribution is generated and plotted in figure 6 together with the original (smoothed) input distribution. This results in a reference interval of $53.8-108.2$ $\mu \mathrm{mol} / \mathrm{l}$ which compares favourably with the reference interval of $53.4-107.6 \mu \mathrm{mol} / \mathrm{l}$ as derived directly.

In table 1 the comparative results of reference intervals for several other constituents are given. It can be concluded that without exception each of these parameters can be processed both with or without transformation of an appropriately selected part of the total distribution. This enables a straightforward and even automatic processing of every result file by firstly looking after an appropriate part of the first derivative plot with a large enough linear portion. In cases of a Gaussian sub-distribution this linearity will frequently be a perfect one and, as was seen in figure 9 for the $\gamma$-glutamyltransferase example, in case of a skewed sub-distribution this linearity is much less perfect, but still enables the location of the boundaries of the region at which the transformation algorithm has to operate. In fact, for Gaussian sub-populations no transformation routine need be applied, but it can be seen from table 1 that there is no disadvantage in doing it. Therefore by deliberately applying the transformation procedure, all distributions can be handled, regardless of their origin.

Tab. 1. Reference intervals deduced either with or without inclusion of the transformation procedure with the function $Z=\ln (x+c)$.

The reference interval consists of the region covered by the 2.5-97.5 percentile limits.

The values shown are for males born between 1940 and 1950.

\begin{tabular}{|c|c|c|c|}
\hline \multirow[b]{2}{*}{ Component } & \multirow[t]{2}{*}{ Unit } & \multicolumn{2}{|c|}{ Reference interval } \\
\hline & & $\begin{array}{l}\text { Without } \\
\text { transformation }\end{array}$ & $\begin{array}{l}\text { After } \\
\text { transformation }\end{array}$ \\
\hline Sodiu & $\mathrm{nol} / \mathrm{l}$ & $136.9-145.7$ & $136.6-146.2$ \\
\hline Potassium & 1 & $3.1-4.7$ & $3.2-4.9$ \\
\hline Chloride & 11 & $98.5-109.5$ & $98.6-109.6$ \\
\hline Bicar & 11 & $21.1-33.1$ & $21.1-33.1$ \\
\hline Calci & 1 & $2.16-2.65$ & $2.17-2.64$ \\
\hline $\begin{array}{l}\text { Inorganic } \\
\text { phosphorus }\end{array}$ & $\mathrm{mmol} / 1$ & $0.57-1.53$ & $0.58-1.5$ \\
\hline Haemoglobin & $\mathrm{mmol} / \mathrm{l}$ & $7.6-11.0$ & $7.7-11.0$ \\
\hline Urea & $\mathrm{mmol} / \mathrm{l}$ & $1.8-9.2$ & $1.8-9.2$ \\
\hline Creatininc & $\mu \mathrm{mol} / \mathrm{l}$ & $53.4-107.6$ & $53.8-108.2$ \\
\hline Total protein & $\mathrm{g} / \mathrm{l}$ & $61.5-82.9$ & $62.0-\$ 3.1$ \\
\hline Cholesterol & $\mathrm{mmol} / \mathrm{l}$ & $2.8-7.9$ & $2.8-7.9$ \\
\hline Uric acid & $\mathrm{mmol} / \mathrm{l}$ & $0.13-0.54$ & $0.13-0.54$ \\
\hline
\end{tabular}


It has been realised that a sound application of the Bhattacharya technique is only feasible when the input distribution i. e. the total hospital population, is homogeneous and stable in time. This means that it is required that the reference intervals deducted from it do not vary too widely. We therefore studied the behaviour with time of the statistical properties of three example files. Table 2 shows the results for creatinine (males), uric acid (males) and alanine aminotransferase (females) sampled in four different calendar periods. It can be concluded that the mean and standard deviation of the input distributions in no case show a larger coefficient of variation than $5 \%$. It is also shown that in spite of the large time interval between the first and fourth calendar period the reference intervals deducted are remarkably constant. This at the same time implies that good analytical precision has been achieved over the two-year period studied.

In table 3 the reference intervals for 15 clinical chemical parameters are listed for both sexes and for 7 age classes. The age classes consisted of people born before 1910, five further decades and finally for people born between 1960 and 1965. The various findings will be briefly discussed.

\section{Serum albumin}

Especially in the adult ages under 50 the concentration in males tends to be 1 to $2 \mathrm{~g} / 1$ higher than in females. Both in males and in females a negative correlation with age with equal regression coefficients of $-0.06 \mathrm{~g} / \mathrm{l}$ is detectable. Reviewing other reported findings, most authors agree with the sex difference $(21-24)$. Both sexes show a more or less constant level after the age of 50 years. For females this finding corroborates with other reported results $(21-23)$.

\section{Serum total protein}

In the adult ages under 50 , the mean serum total protein concentration for males is about $1 \mathrm{~g} / 1$ higher than in females. After the beginning of the menopause the female levels become close to the males with a tendency to be slightly higher. These observations are also reflected in the overall regression coefficients of $-0.06 \mathrm{~g} / \mathrm{l}$ for males and $-0.03 \mathrm{~g} / \mathrm{l}$ for females. All other reports seen by us agree with the reported sex difference $(21,23,24,26-29)$, but not all authors agree about the fall with age.

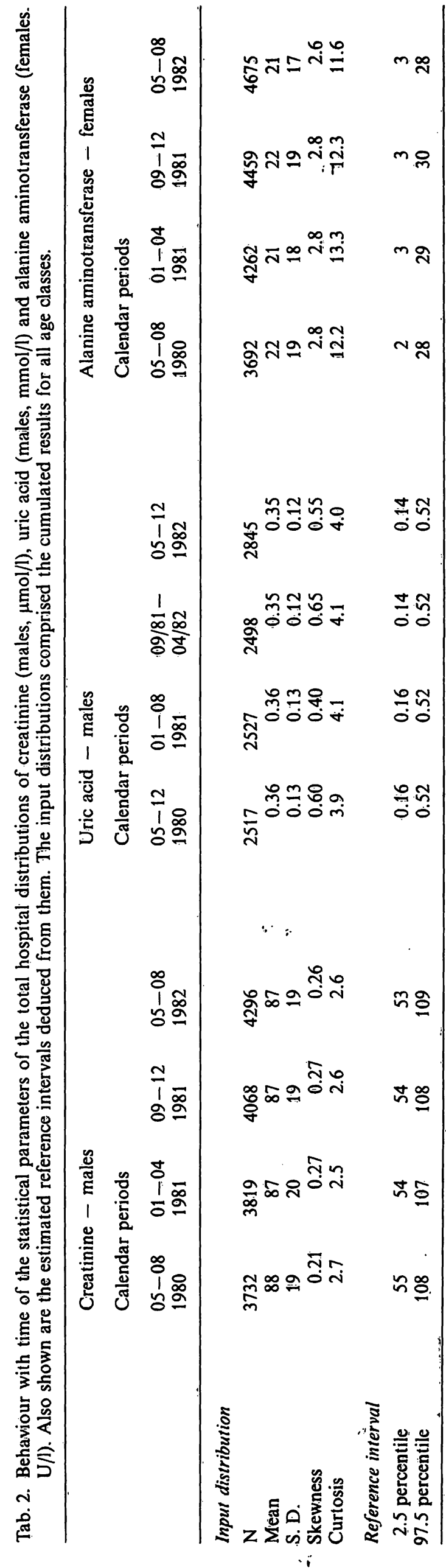

J. Clin. Chem. Clin. Biochem. / Vol. 23, 1985 / No. 12 


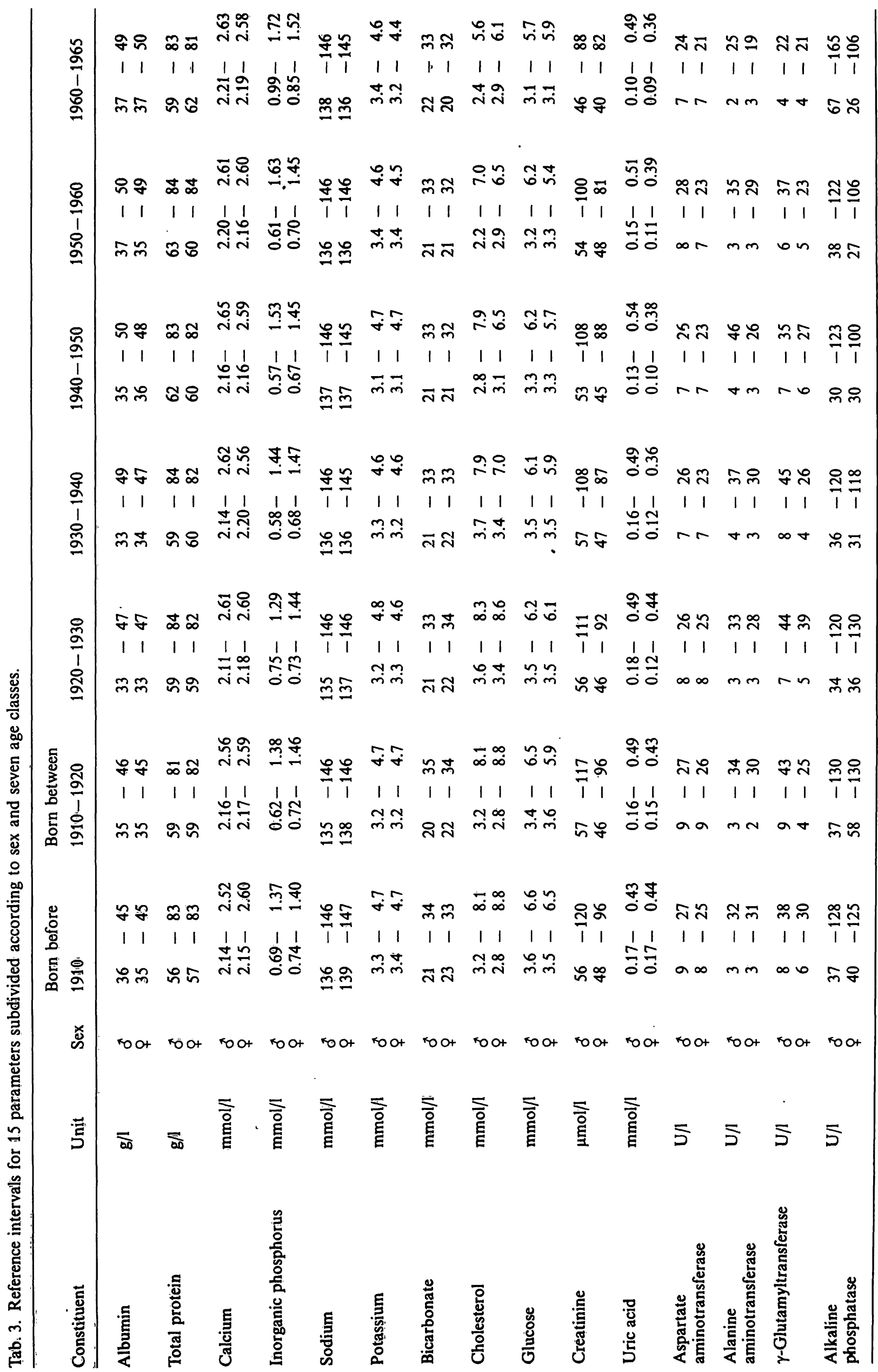




\section{Serum calcium}

For all age periods studied, the mean level of the serum calcium concentration in females is about 2.38 $\mathrm{mmol} / \mathrm{l}$, whereas in men, throughout the age period $20-75$ years, a steady decrease from $2.42 \mathrm{mmol} / 1$ to $2.33 \mathrm{mmol} / 1$ is found. Other reports agree with the female values being less than the male values in the age class $20-50$ years $(23,24,29)$ but some do not find the turnaround phenomenon at the menopausal years $(24,29)$. This is partly caused by the fact that the ages over 50 years were not included in all studies (24). Especially in the ages under 50 years the sex difference is corroborated by a similar difference for serum albumin and total protein. This point has also been focussed upon by Roberts (24).

\section{Serum inorganic phosphorus}

It is apparent that starting from the age of about 30 years the female levels of inorganic phosphorus are about $0.6 \mathrm{mmol} / 1$ higher than the corresponding male levels, a fact that is agreed upon by almost all other authors $(21,23,24,26,29)$. Our data do not confirm an extraordinary difference between the trends of serum inorganic phosphorus in men and women as reported by various authors $(21,22,27)$.

\section{Serum sodium}

Generally, we found no difference between the male and female levels in the pre-menopausal years. Whereas in males no regression with age could be established, the female levels appear to rise after the onset of the menopause. As a result, the mean female levels at the age of 70 years are about $1.5 \mathrm{mmol} / \mathrm{l}$ higher than those found in males. This finding is also reflected by the reports of Wilding et al. (22) and of McPherson et al. (23). Roberts (24) did not find any significant age or sex related difference for serum sodium.

\section{Serum potassium}

We found no clear relationship between age and the serum potassium values. The overall mean values in women $(3.93 \mathrm{mmol} / \mathrm{l}$, range $3.25-4.60 \mathrm{mmol} / \mathrm{l})$ appeared to be somewhat smaller than those in men ( $3.96 \mathrm{mmol} / \mathrm{l}$, range $3.27-4.67 \mathrm{mmol} / \mathrm{l})$. This finding corroborates with the other consulted reports $(22-24)$, although all these papers report a slight positive regression with age.

\section{Plasma bicarbonate}

In men we did not find an age-induced change in the plasma bicarbonate content. The female levels initially are about $1 \mathrm{mmol} / \mathrm{l}$ lower than the corresponding male levels. In the postmenopausal years the female levels rise and have a tendency to be about $1 \mathrm{mmol} / \mathrm{l}$ higher than in males. This is essentially as reported by $M c$ Pherson et al. (23). No other reports were available for comparison.

\section{Serum cholesterol}

In the adult ages till about 50 years the male levels are higher than the corresponding female levels. Both sexes show a positive regression with age until the age of about 60 years $(0.05 \mathrm{mmol} / \mathrm{l}$ per year for men and $0.03 \mathrm{mmol} / \mathrm{l}$ per year for women). The male levels start slightly lower and the initial increase lessens after the years over 40 . Our results also show that after the age of 60 years both sexes show a tendency for lower cholesterol levels, the fall in men being steeper than in women. As a consequence the female levels frequently surpass the male levels by about $0.2-0.5 \mathrm{mmol} / 1$. All studies reviewed by us confirm these cholesterol fluctuations $(22,23,26,29,30)$.

\section{Plasma glucose}

We found a positive correlation with age of about $0.01 \mathrm{mmol} / 1$ per year both for men and women. This means that from the beginning of adult life towards the age of 70 years the mean glucose level rises by about $0.5 \mathrm{mmol} / \mathrm{l}$. From all other reports only the paper of McPherson et al. (23) discusses the age- and sex-related changes of glucose. Whereas they did not find a significant sex-related difference, it was noticed that between 18 and 65 years of age, both sexes show a rise of $10 \%$ in mean glucose levels. This is confirmed by our results.

\section{Plasma creatinine}

The wellknown phenomenon of the sex difference for plasma creatinine levels is easily detectable. It is seen that the mean female levels are about $15 \mu \mathrm{mol} / 1$ lower than the corresponding male levels. This compares favourably with the difference of $16 \mu \mathrm{mol} / \mathrm{l}$ as reported by $M c$ Pherson et al. (23) and of $18 \mu \mathrm{mol} / 1$ as found by Wilding et al. (22). Also shown is the positive regression with age for both sexes. Apart from the late childhood values it can be calculated that in men the rise with age is $0.22 \mu \mathrm{mol} / \mathrm{l}$ per year and for women 
$0.14 \mu \mathrm{mol} / 1$ per year. Although all of the other reports consulted agree about the sex difference there is no consensus about the age-related effects. Whereas Wilding et al. (22) and McPherson et al. (23) agree with our findings, Williams et al. (26) and Roberts (24) found no or at best a slight positive regression with age.

\section{Serum uric acid}

For the adult ages between 20 and 70 years men show a steady mean level of about $0.33 \mathrm{mmol} / \mathrm{l}$. In this same age interval till about the beginning of the menopause, the female levels are always lower than the male levels by about $0.08 \mathrm{mmol} / \mathrm{l}$. Possibly induced by a menopausal effect, the female levels start to rise after the age of about 50 years and finally reach essentially the male levels at the age of about 70 years. Apart from Williams et al. (26), all other reports reviewed describe an age-related effect $(22-24$, $29,31)$. Our findings are exactly reflected by the results of $M c$ Pherson et al. (23).

\section{Serum aspartate aminotransferase}

In men we found no clearcut change with age in the adult age groups. In women a slight positive correlation with age was found. The upper limit for female values, starting at about $4 \mathrm{U} / \mathrm{l}$ lower than the male values at the age of about 20 years, rose to the male level of about $26 \mathrm{U} / 1$ at the age of about 70 years. These findings corroborate with all other reports reviewed $(22,23,26,29)$.

\section{Serum alanine aminotransferase}

The levels of the serum alanine aminotransferase activities throughout life show a remarkable picture for the male values, which peak between $30-40$ years. This phenomenon was only partly be confirmed by the only other report on serum alanine aminotransferase. In this report of Goldberg et al. (32) it was shown that after the age of 30 years the activity in males decreased significantly with age. For females they found, as we did, no consistent change with age, their levels being always less than the corresponding male values.

\section{Serum $\gamma$-glutamyltransferase}

A clearcut difference between male and female levels of serum $\gamma$-glutamyltransferase activity during adult life is discernable. In both sexes we noticed a posi- tively age-related regression until the age of about 50 years. Thereafter this picture changes into a negative age-correlation. The sex difference is confirmed by the reports of Williams et al. (26) and Knight \& Haymond (33). Williams et al. (26) report a steady increase in women till the age of about 56 years. Whether they fail to assess the female decrease at the ages over 60 , because of lack of information from these older age groups, is not clear.

\section{Serum alkaline phosphatase}

Especially in females a positive regression with age from 30-60 years is noticed. This appears to be caused by an increase of the lower reference limits. As also noticed by Goldberg \& Winfield (32), the mean female values reach or surpass the male values after 50 years of age. Particular attention should be payed to the large mean value and upper reference limit in boys in the age group 17-21 years. As noticed by others $(23,33,34)$, the initial high childhood values, with higher values in boys than in girls, level off in boys after the age of 18 years, whereas in girls the decline towards adult values starts at the age of 17 years. This is also reflected by our high reference interval in the male category in the age group 17-21 years (mean 105, upper limit $165 \mathrm{U} / \mathrm{l}$ ) compared with the next male age group of $21-30$ years (mean 80 , upper limit $122 \mathrm{U} / \mathrm{l})$.

\section{Discussion}

Interpretation of the result of a biochemical test requires to answer the question of whether or not the test result belongs to the distribution of values found in a particular population. The composition of this target population can vary according to the interest of the intended user. This necessitates the accurate definition of the members of the target population (29). Further, the conditions under which the analytical samples are collected and processed have to be stringently standardised. In spite of the criticism that has been raised about the use of the so-called indirect methods for estimating reference intervals (3, $36-38$ ), we thought that it remained an attractive alternative approach in view of the practical difficulties just mentioned. Moreover, in hospital, blood specimens are frequently collected under a wide variety of conditions which, without doubt, have effect on the final analytical results. Therefore, reference intervals deducted from selected healthy samples, with use of state of the art procedures, may not 
provide the information needed to make the appropriate clinical decision, because what is gained in sensitivity is lost in specificity. By using as input the total bulk of test results from all admitted patients, one automatically obtains a one to one relationship between the selected population sample and the category of people for which the test results are intended.

The application of the Bhattacharya deconvolution technique of partly overlapping Gaussian distributions is hampered by the restriction that the method becomes invalid when the composite distributions are too close to each other, thereby preventing the recognition of the linear part in the first derivative function. It is easily imagined that in a situation where all hospital admissions are followed by a routine screening of a biochemical test profile, this requisite is much more easily fulfilled, because the proportion of normal values is increased in comparison with the situation where such a routine screening procedure is absent, and where the laboratory mainly provides answers to more or less specific requests.

In our view, this drawback will not prevent the application of this technique when it is properly used. We have therefore included the restriction that result files may only be processed when a large enough linear part of the first derivative function, in terms of the percentage of the total population, can be found. In practice we have always set this restrictive boundary at $40 \%$.

The merits of indirect estimation procedures are often criticised on the basis that hospital populations by nature are heterogeneous with no discernable main population with any stability or reproducibility. We believe we have presented evidence (tab. 2) that with a large enough population sample the stability of the total hospital population is satisfactory. Together with the above-mentioned restriction of $40 \%$, this gives enough assurance for a sound application.

A further argument in favour of this technique is the agreement with the observations reported by other authors on the age- and sex dependency of reference intervals. It has to be realised that often these relationships are relatively weak. Nevertheless this technique appears to be able to resolve such weak relationships.

We therefore believe that with this relatively easy technique every laboratory is able to assess its own reference intervals, divided, when possible, according to sex and age, and according to the local conditions.

\section{References}

1. Schütz, W. (1984) Lab. Med. 8, 212-219.

2. Sunderman, F. W. (1975) Clin. Chem. 21, 1873-1877.

3. Elveback, L., Guillier, C. \& Keating, F. (1970) J. Amer. Med. Assoc. 211, 69-75.

4. Mainland, D. (1969) Ann. N. Y. Acad. Sci. 161, 527-537.

5. IFCC Expert Panel on Theory of Reference Values (1979) Clin. Chem. 25, 1506-1508.

6. IFCC Expert Panel on Theory of Reference Values (1983) J. Clin. Chem. Clin. Biochem. 21, 749-760.

7. Grasbeck, R. \& Solberg, H. E. (1981) Clin. Chem. 27, 1795- 1796.

8. White, J. D. (1978) Clin. Chim. Acta 84, 353-360.

9. Gindler, E. M. (1970) Clin. Chem. 16, 124-126.

10. Naus, A. J., Borst, A. \& Kuppens, P. S. (1980) J. Clin. Chem. Clin. Biochem. 18, 621-625.

11. Naus, A. J. (1982) M. D. Thesis, Maastricht, The Netherlands, 1982.

12. Bhattacharya, C. (1967) Biometrics 23, 115-135.

13. Baadenhuijsen, H., Arts, J., Somers, L. \& Smit, J. C. (1984) REFVALUE, A Software Package to calculate reference intervals from total hospital patient laboratory data. ISBN: 0.444-42322-2, Elsevier Science Publishers, Amsterdam, New York, 1984.

14. Lilliefors, H. (1967) J. Amer. Statist. Assoc. 62, 399-402.

15: Shapiro, M., Wilk, M. \& Chen, H. (1968) J. Amer. Statist. Assoc. $631343-1372$

16. Solberg, H. E. (1981) In: Reference Values in Laboratory Medicine (Grasbeck, R. \& Alstrom, T., eds.) pp. 193-205, John Wiley and Sons, Chichester.
17. Boyd, J. C. \& Lacher, D. A." (1982) Clin. Chem. 28, $1735-1741$

18. Harris, E. K. \& DeMets, D. L. (1972) Clin. Chem. 18, $605-612$.

19. Manly, B. F. J. (1976) The Statistician 25, 37-42.

20. Savitsky, A. \& Golay, M. (1964) Anal. Chem. 36, $1627-1638$.

21. Keating, F. R., Jones, J. D. Elveback, L. R. \& Randall, R. V. (1969) J. Lab. Clin. Med. 73, 825-834.

22. Wilding, P., Rollason, J. G. \& Robinson, D. (1972) Clin. Chim. Acta 41, 375-387.

23. McPherson, K., Healy, M. J. R., Flynn, F. V., Piper, K. A. J. \& Garcia-Webb, P. (1978) Clin. Chim. Acta 84, $373-397$.

24. Roberts, L. B. (1967) Clin. Chim. Acta 16, 69-78.

25. Wingerd, J. \& Sponzilli, E. E. (1977) Clin. Chem. 23, $1310-1317$.

26. Williams, G. Z., Widdowson, G. M. \& Penton, J. (1978) Clin. Chem. 24, 313-320.

27. Werner, M., Tolls, R. E., Hultin, J. V. \& Mellecker, J. (1970) Z. Klin. Chem. Klin. Biochem. 8, 105-115.

28. Reed, A. H., Cannon, D. C., Winkelman, J. W., Bhasin, Y. P., Henry, R. J. and Pileggi, V. J. (1972) Clin. Chem. 18, $57-66$.

29. Goldberg, D. M., Handyside, A. J. \& Winfield, D. A. (1973) Clin. Chem. 19, 395-402.

30. Steinmetz, J., Panek, E. \& Siest, G. (1980) Clin. Chem. 26, 219-226.

31. Petitclerc, C. (1975) Clin. Chem. 21, 1008. 
32. Goldberg, D. M. \& Winfield, D. A. (1974) Clin. Chim. 36. Amador, E. \& Hsi, B. P. (1969) Amer. J. Clin. Pathol. 52, Acta 54, 357-368.

538-546.
53si, B. P. (1969) Amer. J. Clin. Pathol. 52,

33. Knight, J. A. \& Haymond, R. E. (1981) Clin. Chem. 27, 37. Richardson, R., Setchell, K. \& Woodman, D. (1972) Clin.
Ch-51. 48-51.

34. Eastman, J. R. \& Bixler, D. (1977) Clin. Chem. 23, 38. O'Halloran, M., Studley-Ruxton, J. \& Wellby, M. (1970) $1769-1770$

35. Leonard, J. V. \& Westlake, A. J. (1978) Clin. Chim. Acta 82, 271-279. Clin. Chim. Acta 27, 35-46.

Dr. H. Baadenhuijsen

Laboratory of Clinical Chemistry

Department of Internal Medicine

St. Radboud University Hospital

Geert Grooteplein Zuid 8

P. O. Box 9101

NL-6500 HB Nijmegen 
